Thorax (1952), 7, 240.

\title{
CARCINOMA OF THE MIDDLE LOBE
}

\author{
BY \\ P. E. BALDRY \\ From Harefield Hospital, Middlesex
}

(RECEIVED FOR PỤbliCATION NOVEMBER 2, 1951)

The stem bronchus of the middle lobe is, in comparison with other parts of the bronchial tree, an uncommon site of origin for carcinoma. When carcinoma does occur in this site, it often presents diagnostic difficulties, as its clinical picture is very similar to that seen in post-infective bronchostenosis. The latter term will be used to describe the bronchial obstruction brought about either by external pressure from tuberculous lymph nodes, and at times by erosion of these nodes into the bronchial lumen (post-tuberculous bronchostenosis), or by pressure on the bronchus from enlarged infected non-tuberculous lymph nodes, or more rarely by oedema and inflammation of the bronchial wall, without evidence of lymphadenitis. Attention has recently been directed to these types of obstruction by Brock, Cann, and Dickinson (1937), Brock (1943, 1946, and 1950), Graham, Burford, and Mayer (1948), and Paulson and Shaw (1949). Each of these conditions may, by occlusion of the stem bronchus, lead to collapse of the middle lobe with consolidation or even abscess formation, and later bronchiectatic changes. Diagnosis in such cases is not always easy, but is important because they do not present the same urgent indications for surgical treatment as do cases of bronchial carcinoma.

At Harefield Hospital during the past five years nine cases of carcinoma arising from the middle lobe bronchus have been treated by resection. They constituted $5 \%$ of the total of 185 cases in which lung resection for malignant disease was performed during this period (Table I). Each of these nine cases presented diagnostic difficulties, which it is the purpose of this paper to discuss.

TABLE I

Sites of Origin of Bronchial Carcinomata Treated BY RESECTION FROM JULY, 1946, TO JUNE, 1951

\begin{tabular}{|c|c|c|c|c|c|c|}
\hline \multicolumn{3}{|c|}{ Right } & \multirow[b]{2}{*}{$\begin{array}{r}32 \\
9 \\
40 \\
13\end{array}$} & \multicolumn{2}{|l|}{ Left } & \multirow[b]{2}{*}{$\begin{array}{r}42 \\
5 \\
38 \\
6\end{array}$} \\
\hline $\begin{array}{l}\text { Upper lobe } \\
\text { Middle ", } \\
\text { Lower ", } \\
\text { Main bronchus }\end{array}$ & $\begin{array}{l}\cdots \\
\cdots \\
\cdots\end{array}$ & $\begin{array}{l}\cdots \\
\therefore \\
\cdots\end{array}$ & & $\begin{array}{ll}\text { Upper lobe } & \ldots \\
\text { Lingula } & \ldots \\
\text { Lower lobe } & \ldots \\
\text { Main bronchus }\end{array}$ & $\begin{array}{l}\cdots \\
\cdots \\
\cdots\end{array}$ & \\
\hline Total & $\ldots$ & $\ldots$ & 94 & Total & $\ldots$ & 91 \\
\hline
\end{tabular}

\section{HISTORY}

The history was not very helpful, as the syrifptoms in these cases were very similar to those found in patients with post-infective stenosis, and included pain in the chest, cough, sputwo, haemoptysis, and fever. Febrile attacks werष्ठ at times mild, transitory, and repeated at intervals, but more often presented as bouts of acute " pno monia." - It might be thought that the duration of symptoms would be some guide to the deognosis, and that symptoms present for some years would be indicative of a post-infective stenogis. However, carcinoma of low malignancy may a cause symptoms over a long period of time, ôs Cases 1 and 2 illustrate.

Case 1.-A woman aged 33 was well until Januây, 1945, when she began to suffer from cough, wh $\overrightarrow{\mathrm{B}} \mathrm{ch}$ persisted, together with increasing shortness of breath. $A$ radiograph at that time showed a round shadowein the right side of the chest (Fig. 1a). She was keppt under observation until December, 1946, when she was admitted to hospital with right basal pneumo ia and pleurisy. She was treated with penicillin, Eut after recovery from her symptoms a radiograph showed a persistent shadow at the right base. Sुhe was referred to Harefield Hospital in January, 1947. Bronchoscopy revealed a neoplasm in the right mâin bronchus, and a biopsy showed polygonal and columnar-celled carcinoma. Radiographs taken $\mathrm{O}_{\mathrm{J}}$ t this time are shown in Figs. $1 b$ and $c$. On February 6, 1947, right middle and lower lobectomy was formed. The pathologist's report on the removied lobes is as follows:

"The main bronchus is partly obstructed by a snout of growth projecting from the middle lobe, ostifin. The proximal half of the middle lobe containswa spheroidal tumour $4 \times 4 \times 2 \frac{1}{2} \mathrm{~cm}$. which reaches 零e pleural surface. The distal half of the lobe is epllapsed and shows mucus distension of the brontक्षhi. Right lower lobe appears normal.

"The tumour is partly a polygonal-cell trabecutar and partly mucus-secreting tubular carcinoma."

Case 2.-A man aged 40 , in 1945, four years bef(are admission to this hospital, had pneumonia aind pleurisy, and was in hospital for 10 weeks. Affer this he had recurrent pain in the right side of the 


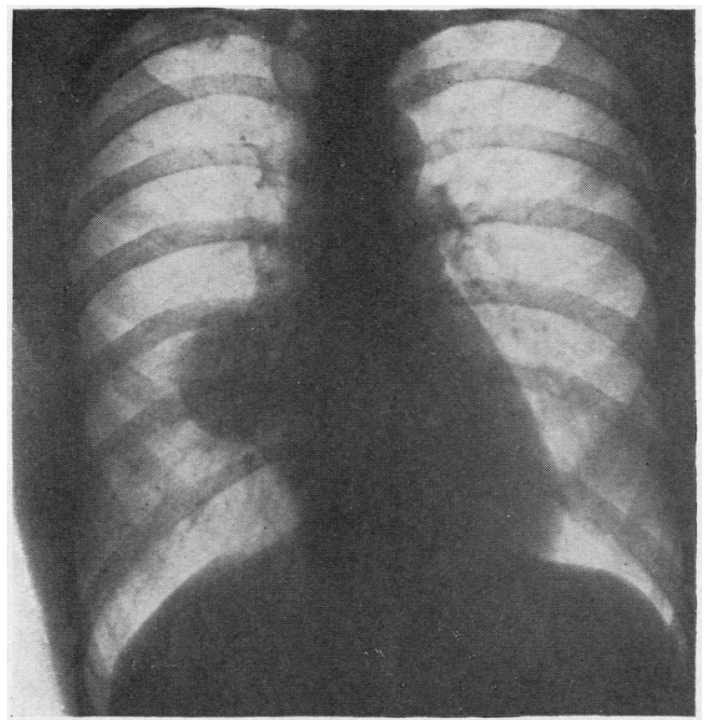

FIG. 1a.-The radiograph shows an opacity which later proved to be a carcinoma arising from the middle lobe bronchus.

chest, coming on every few weeks, lasting about a week at a time, and aggravated by wet weather. There was a slight cough and trace of sputum, but no haemoptysis and no dyspnoea. In January, 1949, he was admitted to Harefield Hospital, feeling fit and putting on weight ; his only complaint was the recurrent pain. He looked healthy, and the only abnormal physical sign was slight diminution of chest movement on the right side.

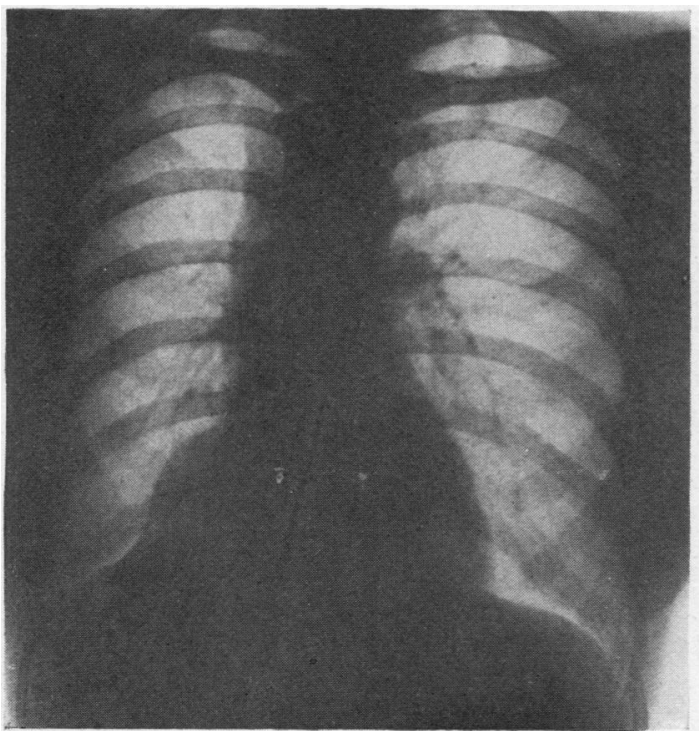

A radiograph at this time showed collapse of the middle lobe. No tubercle bacilli were found in the sputum. Bronchoscopy showed a granulating mass in the floor of the middle lobe bronchus, which bled readily on touch. A biopsy specimen of this was reported as showing a mucus-secreting carcinoma.

On March 14, 1949, right middle and lower lobectomy was performed. The pathologist's report on these lobes was:

"The middle lobe bronchus at its bifurcation is obstructed by a growth measuring about $2.7 \times 1.5 \times$ $1.5 \mathrm{~cm}$. The middle lobe is shrunken, indurated, and adherent, while the lower lobe appears normal.

"A mucus-secreting polygonal carcinoma. The surface of the growth (like the mucosa of the obstructed bronchus) is chronically inflamed, papillated, and covered with hypertrophic ciliated epithelium. The obstructed lung shows fibrosis, pseudoglandular change, and large deposits of cholesterol."

Haemoptysis is a common feature in both postinfective and neoplastic cases, and in the latter may have been recurrent over a long period of time, as is illustrated by Case 3 .

Case 3.-A man aged 40, in 1926, 20 years before admission to this hospital, had a slight haemoptysis. In 1929 he was confined to bed for one week with right-sided pleurisy. Since then he had coughed up blood about once a year.

In April, 1946, he was admitted to Harefield Hospital. A radiograph showed a mass extending from the right hilum. Bronchoscopy at this time showed pus issuing from the middle lobe orifice, which was occluded by a neoplasm with a necrotic and bleeding surface. A biopsy showed tubular carcinoma, possibly of low grade of the type designated adenoma.

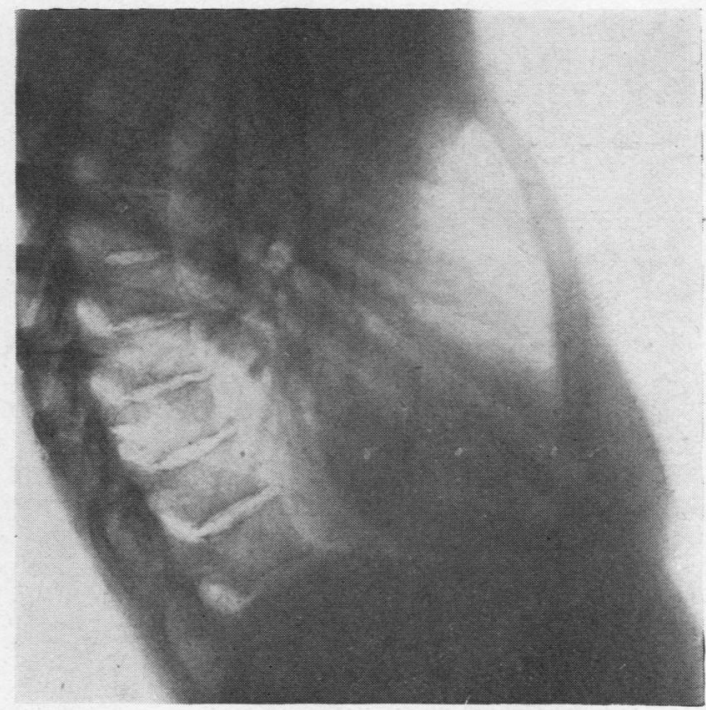

FIGs. $1 b$ and $c$.-Radiographs taken two years later show collapse of the lobe, with distension of the proxima! half by the tumour. 
In August, 1946, right middle lobectomy was performed.

The pathologist's report on this lobe was as follows:

"Polygonal-celled carcinoma; the growth is arranged in trabeculae and interlacing sheets, with scanty mitosis. Infiltration is definite, but the growth is of low malignancy."

Sputa were examined in all cases to exclude the presence of tubercle bacilli, but examination for cancer cells was not at that time a routine procedure in this hospital, and only in one case were the secretions obtained at bronchoscopy examined for malignant cells. The procedure is now more frequently adopted, as it is often of value in cases where bronchoscopy is negative. Woolner and McDonald (1950), in a series of 77 cases of bronchial carcinoma treated by resection, found that in 45 bronchoscopic biopsies before operation and in the remainder examination of sputa or bronchial secretions gave histological confirmation of the diagnosis. The usefulness of cytological examination of the aspirated bronchial secretions is confirmed by the following case.

Case 4.-A man aged 45, three and a half months before admission to this hospital, began to have a dry cough. Two months later he developed pain in the right side of the chest, and coughed up blood-stained sputum for four days.

On admission in April, 1950, he had a large rightsided pleural effusion. Cytological examination of this fluid showed red cells only, and culture was sterile. No tubercle bacilli were seen, found directly, or by culture in the sputum. A radiograph after aspiration of the pleural fluid showed a shadow in the right mid-zone.

Bronchoscopy had been performed before admission, and no abnormality seen, but the bronchial secretions aspirated at this time were sent for special examination for malignant cells.

Bronchoscopy was repeated after admission, and again nothing abnormal was seen. However, by this time the report on the bronchial secretions was received, and this showed that the aspirated fluid contained pus cells, erythrocytes, and numerous cells of malignant type. In view of these findings a right pneumonectomy was performed.

The pathologist's report on the specimen was:

"There is a growth arising from the beginning of the middle lobe bronchus, the lumen of which is reduced to a narrow slit. The growth extends into the adjacent tissue, producing a mass, $2.5 \mathrm{~cm}$. in diameter, about the origin of the bronchus. It extends outwards for about $5 \mathrm{~cm}$. along the obstructed branches of the bronchus and to within $1 \mathrm{~cm}$. of the lateral surface. Beyond the growth there is retention bronchiectasis: mucus distending the bronchi is clear, and lipoid pneumonitis is absent. The contiguogs lymph nodes only appear to be affected.

"Oat-celled carcinoma: the adjacent glands almost completely replaced by growth."

\section{Differential Diagnosis}

Obstruction of a bronchus, whether it is by poseinfective bronchostenosis or by neoplasm, causes collapse of the lung beyond, and before lowg infection gives rise to consolidation or abscests formation, and, if the pleura becomes involved, 化 pleural effusion or empyema. These changes lead to clinical and radiological features which a se especially liable to lead to confusion when the middle lobe is involved.

INTERLOBAR EFFUSION.-There were two casegs in this series in which a consolidated middle lobe, in one case surrounded by pus, and in the other containing pus, was misdiagnosed initially as primary interlobar empyema. The diagnosis hat been made in each case because of the appearange in a lateral radiograph of a circumscribed ov s. shadow with bulging edges lying along the like usually occupied by the lower end of the greater fissure, and also because pus had been aspirated from the area. After further analysis, however, $\overline{\mathrm{Bf}}$ the appearances in the postero-anterior as well ofs the lateral films, the correct interpretation was apparent.

"Primary" interlobar empyema is rare and the end-result of a generalized pleural infection, the fluid from the main pleural space having absorbed, leaving a residual pocket in one of the fissures. At this stage, the, underlying lung disease has usually resolved, and it is safe to treât the empyema as the principal condition. Moge frequently, a localized interlobar empyema secondary to concurrent disease in the adjacent lung. It commonly arises as a result of rupture of a lung abscess (Neuhof and Copleman, 1940). In addition, pus may surround a collapsed a consolidated middle lobe, and this is apt to leat to confusion, as the shadow produced has certas features in common with those of an interlobar empyema. This difficulty arose in Cases 5 and 8 .

Case 5.-A man, aged 56, had had bronchitis $\mathrm{f}^{\mathrm{W}}$ years, mostly in the winter, coughing up mucopus, and had been becoming progressively more short of bre and wheezy. Two months before admission he cosulted his doctor about an attack of "fibrositis" of the left thigh and, in passing, mentioned that he hag recently developed a pain in the right side of the che?, worse on taking a deep breath, and that his cough and expectoration were worse than usual. He fot ill and was feverish, and had lost $14 \mathrm{lb}$. (6 kg.) 용 weight in the past year. 

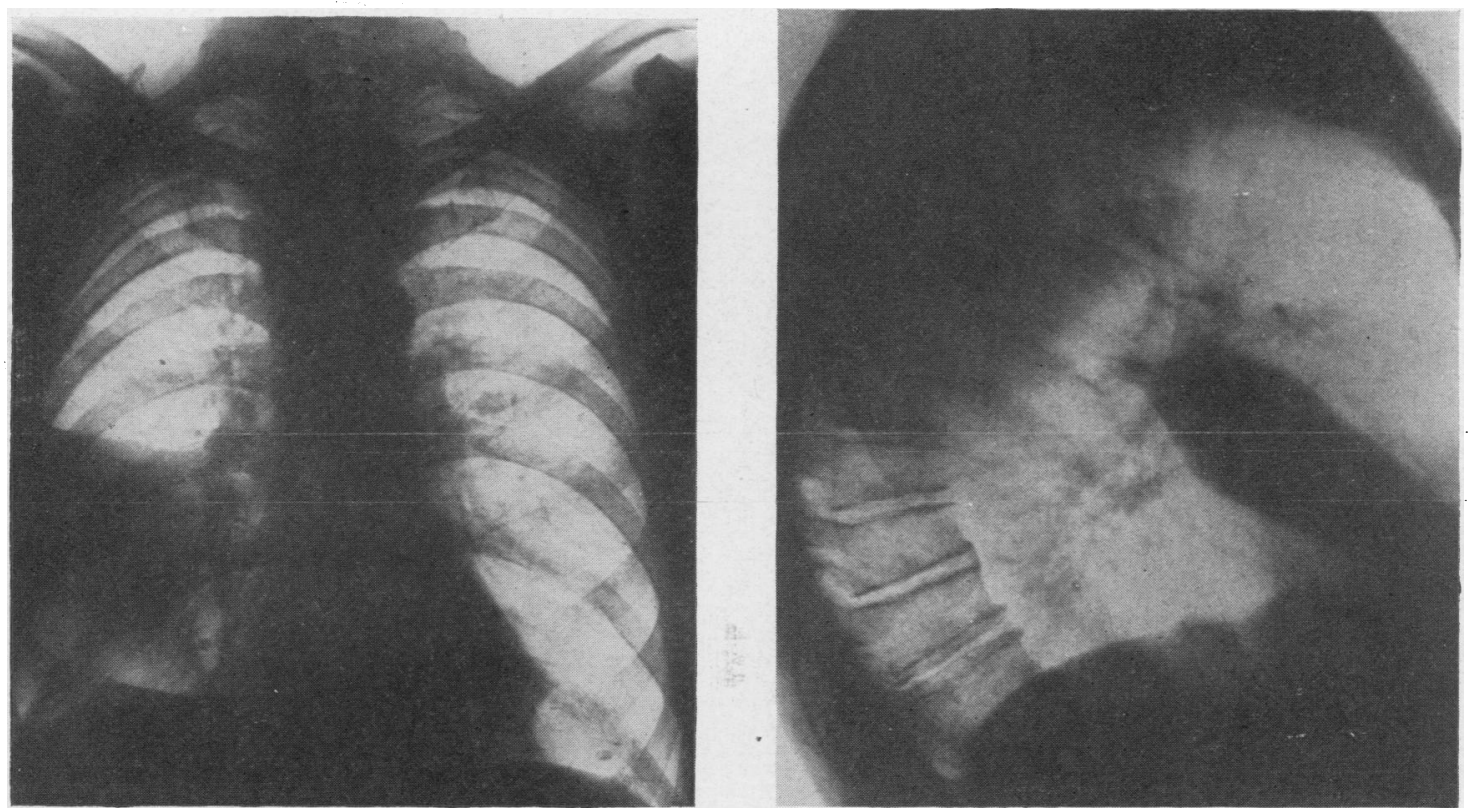

Figs. $2 a$ and $b$.- Radiographs show collapse and consoiidation of the middle lobe as a result of a carcinoma occluding the stem bronchus. There was a collection of pus around this lobe, and this finding, together with the radiographic appearances, led at first to a mistaken diagnosis of primary interlobar empyema.

Radiographs (Figs. $2 a$ and $b$ ) of the chest were thought to indicate an interlobar empyema, and on needling $4 \mathrm{oz}$. of thick pus was withdrawn. This contained polymorphs and debris, but on culture was sterile. Bronchoscopy was normal, and he was therefore transferred in January, 1951, to Harefield Hospital as a case of encysted empyema for treatment. Bronchoscopy was repeated and was negative. At thoracotomy there was considerable thickening of the pleura underlying the incision, with dense adhesions. Aspiration deep to this produced thick, purplish pus. On incising the pleura a little pus was discovered in the interlobar fissure. The middle lobe was found to be enlarged and consolidated, secondary to a growth arising from its stem bronchus, and, after some difficulty owing to adhesions, it was removed.

The pathologist's report on this specimen was as follows:

"The lobe is ragged from adhesions, and shows a growth in the cut hilar surface. The growth, which measures $4.5 \times 3.5 \times 5 \mathrm{~cm}$., is largely necrotic. Beyond the growth the lung shows retention bronchiectasis, with ulceration, and lipoid pneumonitis with fibrosis.

"The growth is a squamous-celled carcinoma with scirrhous fibrosis and moderate keratinization. Adjacent lymph glands at the hilum are unaffected."

Case 6.-A woman, aged 48, had been well until four months before admission, when she developed a cough with 2 to $3 \mathrm{oz}$. (60-90 ml.) of mucoid sputum a day. Six weeks later she developed a pleuritic pain in the right side of the chest, which lasted for about one week and was treated by rest in bed. On January
23, 1951, a radiograph is said to have shown a right interlobar effusion.

She was admitted to a hospital and treated with 900,000 units of penicillin twice a day for a fortnight. Aspiration of the chest produced $40 \mathrm{ml}$. of pus growing pneumococci, and a diagnosis of right interlobar empyema was made.

She was transferred to Harefield Hospital on March 12, 1951. Radiographs (Figs. $3 a$ and $b$ ) showed a shadow in the position of the middle lobe, consistent with obstruction and distension of this lobe. Bronchoscopy showed a mass protruding from the middle lobe orifice. Biopsy of this was reported as showing squamous-cell carcinoma.

On April 3, 1951, right pneumonectomy was performed. No pus was found lying free in the pleural cavity. The appearance of the middle lobe is shown in Fig. 4. The straight upper edge formed by the boundary of the lesser fissure which had been noticed on the postero-anterior radiograph is clearly shown.

The pathologist's report on the specimen was as follows :

"The middle lobe bronchus is occluded by growth about $1.3 \mathrm{~cm}$. beyond its origin, reducing the lumen to a slit. The growth extends into the lobe, forming a mass $7.5 \times 7.5 \times 4.5 \mathrm{~cm}$. It reaches the surface of the specimen medially and extends for $2.2 \mathrm{~cm}$. into the upper lobe. Beyond the growth there is chronic bronchiectasis with lipoid pneumonitis and fibrosis. Some of the dilated bronchi have a lining of lipoid histiocytes. Parts of the growth are necrotic: these are pultaceous and creamy yellow, while the nonnecrosed parts are greyish. 

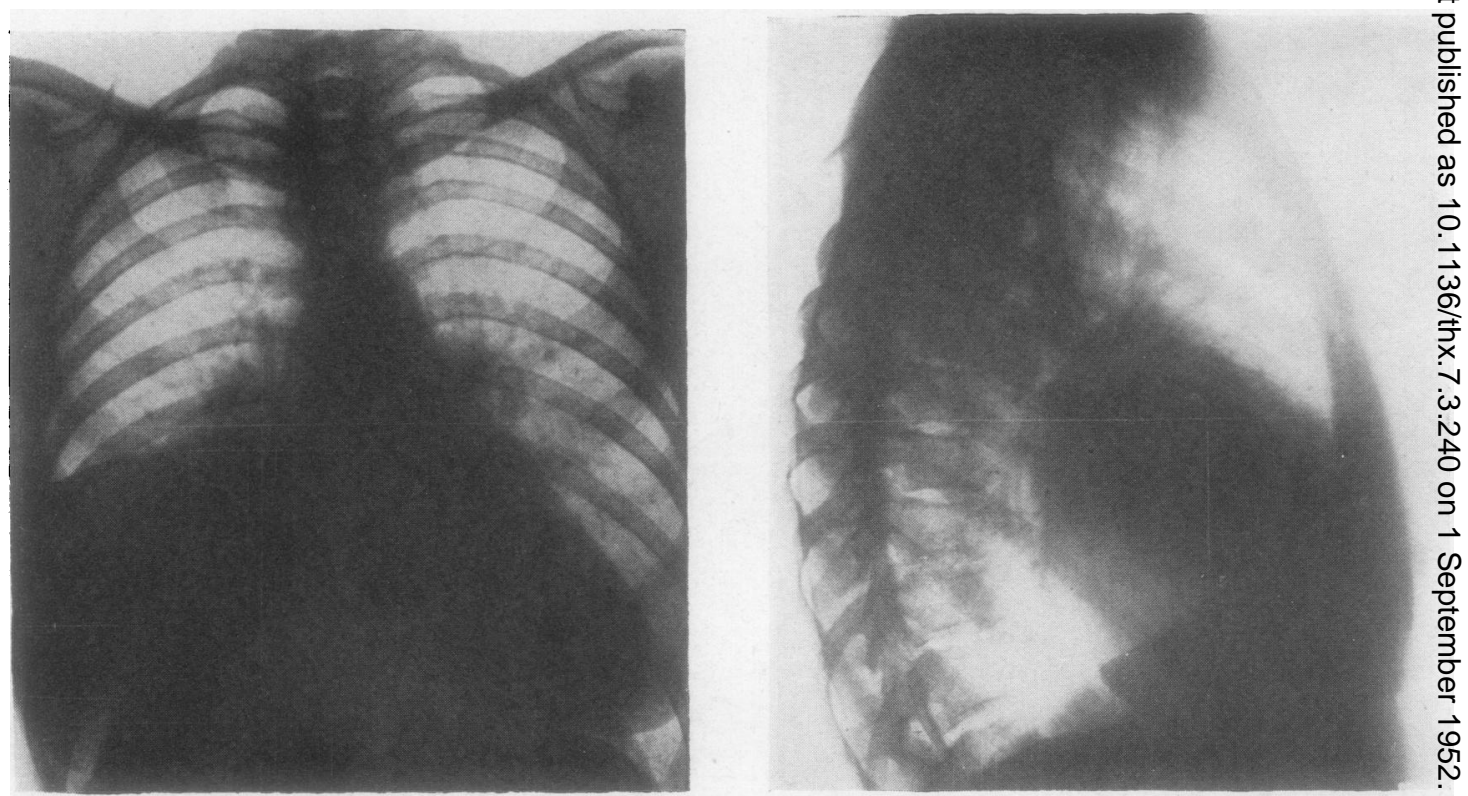

Figs. $3 a$ and $b$-Radiographs show collapse and distension of the middle lobe brought about by a carcinoma which arose from the stem bronchus and then widely invaded the lobe. Pus aspirated from the area led initially to the erroneous view that the appearances were due to an interlobar empyema.

" This is a typical squamous carcinoma with moderate keratinization and scirrhous fibrosis. The anterior hilar glands are involved."

In retrospect it would seem that the pus aspirated from this case was withdrawn from the breaking-down necrotic growth itself.

The radiographs of these two cases show in each instance an opacity in the postero-anterior view bounded above by a horizontal line which was clearly running along the line of the lesser fissure. If, however, the shadow had been due to effusion into this fissure, it would have appeared higher up on the lateral film, and not, as it did, extending down to the position usually occupied by the lower part of the greater fissure. Effusion into the greater fissure does not have a horizontal upper border in the postero-anterior view, but usually gives rise to a globular shadow or a shadow with an ill-defined upper margin. It was clear, therefore, that the opacity could not be due solely to effusion into either fissure. This was supported by the observation that in both these cases neither fissure could be seen running separately from the main shadow, whereas in a case where there is effusion into one fissure the other fissure shows in its normal position. The opacity being produced principally by a collapsed and consolidated middle lobe, the fissures formed the upper and lower boundaries of it. Other points against the diagnosis of primary interlobar empyema were the absence of a generalized adhesive pleuritis, except that in Case 5 peaking of the right side the diaphragm could be seen, and the normet appearance of the posterior part of the greate fissure, since in cases of effusion into the anteriö part of the fissure there is usually thickening also of the upper part.

LUNG ABSCESS.-Carcinoma of the lung no infrequently presents as a lung abscess. Masom (1949) found that $10 \%$ of a series of 1,000 cas of bronchial carcinoma presented with a cavity

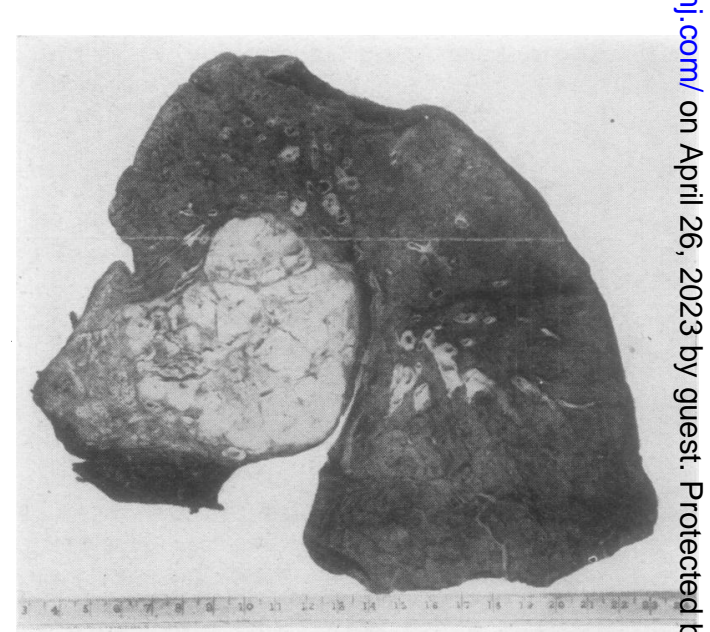

FIG. 4.-Photograph showing the middle lobe extensively replaced by growth. 
and fluid level. However, if by careful radiology such a lesion is localized to the middle lobe, it is more likely to be part of the post-tuberculous syndrome (Brock, 1950). Nevertheless, it may be secondary to a neoplasm blocking the middle lobe bronchus, or actually be formed by necrotic neoplastic tissue in that lobe. Case 7 illustrates this point.

Case 7.-A man, aged 62, in January, 1950, slipped at work and hit the right side of his chest. He had had a morning cough and sputum for years, and three weeks after this accident he noticed streaks of blood in his sputum. After another week he coughed up. on two successive days, an egg-cupful of foultasting, blood-stained sputum "as if coming from an abscess."

On March 10, 1950, he was admitted to Harefield Hospital. His general condition was fair. Soft enlarged lymph nodes were felt in the right axilla and one above the clavicle. Breath sounds were weak, with many rhonchi, in the lower part of the right side of the chest anteriorly. Radiographs (Figs. 5a and $b$ ) showed an opacity in the middle lobe, more or less rounded, with excavation and a fluid level.

Sputum examined on several occasions showed no tubercle bacilli.

Bronchoscopy showed pus coming from the middle lobe orifice, but no evidence of stenosis.

Penicillin, 2,000,000 units a day, was given for a fortnight, but as there was no radiological improvement a thoracotomy was performed on April 25, 1950 , and the middle lobe removed, together with the lower part of the upper lobe.

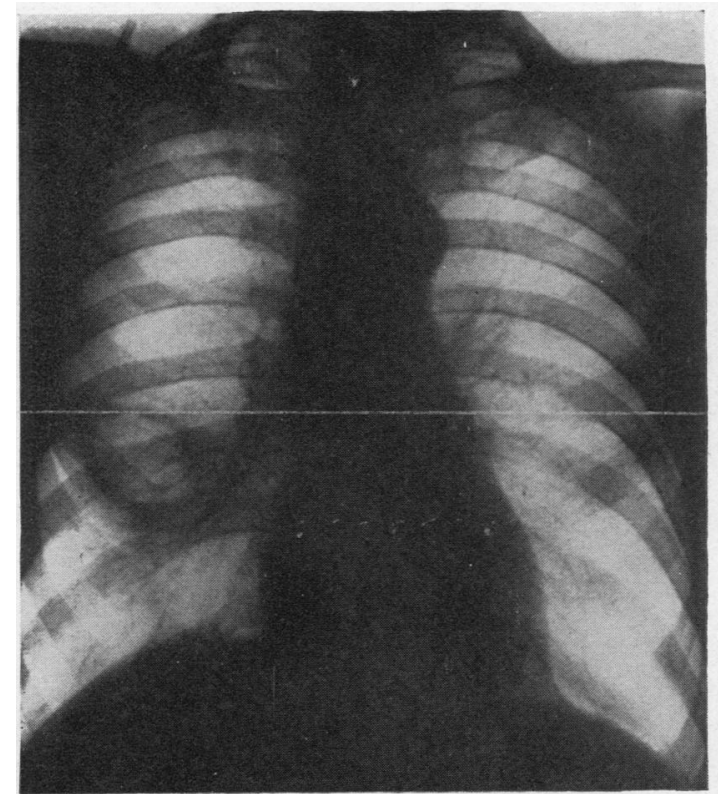

The pathologist's report on the specimen was as follows :

"The middle lobe contains a large cavernous growth, $3.4 \mathrm{~cm}$. from the cut end of the bronchus. The cavity, which was about $4 \mathrm{~cm}$. in diameter, was ruptured on the medial aspect of the specimen.

"This is a squamous-celled carcinoma with welldifferentiated prickle cells and some keratinization."

The Significance of Calcification.-In middle lobe disease radiographic evidence of calcification at the hilum is generally regarded as a strong indication that the changes arise from post-tuberculous stenosis of the bronchus; but by no means all cases of this condition show obvious calcification on the radiograph, and, on the other hand, the presence of calcification should not be taken as final proof of the tuberculous basis of a middle lobe lesion. It is possible that a patient who has had a heavy primary tuberculous infection with residual calcification of the hilar glands may develop a carcinoma arising from the middle lobe bronchus. In Case 8, to be described below, a carcinoma of the middle lobe bronchus occurred in a patient whose radiograph showed old-standing tuberculous disease with fibrosis and calcification in the right upper zone, and, as bronchoscopy was negative, it would have been very easy to have assumed that the consolidation in the lateral segment of the middle lobe was also related to the tuberculous infection.

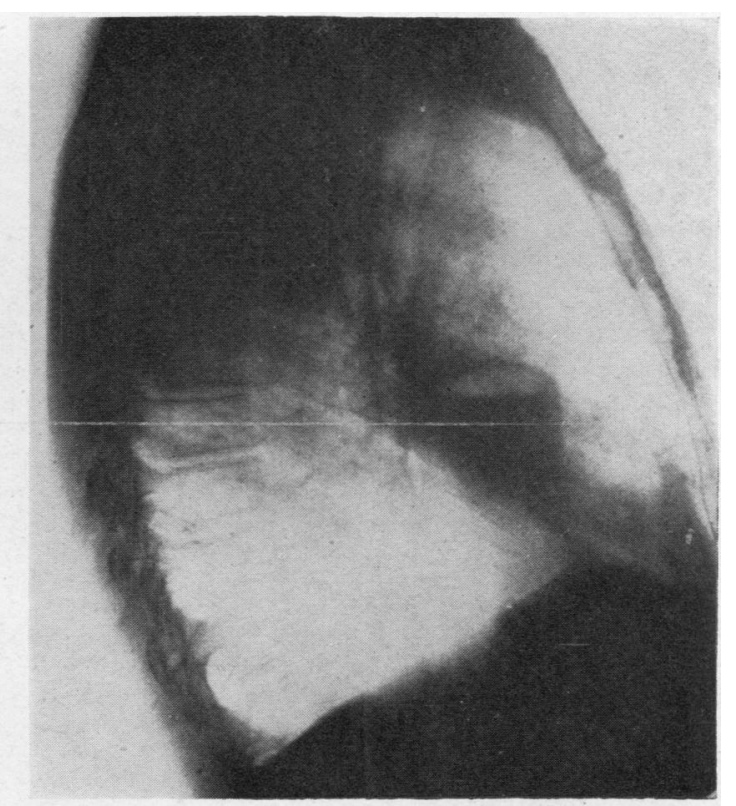

FiGs. $5 a$ and $b$. - Radiographs showing an "abscess" in the middle lobe which proved to be a breaking-down neoplasm. 
BRONCHOGRAPHY.-This investigation is worth performing if only to demonstrate the block which is the cause of the collapse and inflammatory changes seen in the middle lobe itself, but it does not as a rule give any clue to the nature of the obstruction. Touroff (1949), however, has stated that at times he has observed an area of bronchial narrowing $\frac{1}{2}$ in. or more in length in cases of postinfective stenosis, in distinct contrast to the complete occlusion or short area of bronchial narrowing that is usually seen in carcinoma.

BRONCHOSCOPY.-This investigation is unfortunately not always as helpful as one might expect, and at times is misleading. Carr, Skinner, Robbins, and Kessler (1950) in a review of their cases estimated that only about $40 \%$ of all bronchial tumours originated in a part of the bronchial tree where they could be seen directly by bronchoscopy and a biopsy taken. An additional source of error arises when abnormal tissue is seen, and on biopsy proves to be granulation tissue, suggesting a primary inflammatory process, whereas in fact inflammation is secondary to a distal carcinoma. Case 8 illustrates this difficulty.

Case 8.-A man aged 54 had had slight morning cough and sputum with shortness of breath on exertion for some years.

In October, 1948, he had a sudden pain in the right lower chest, and this pain persisted until January, 1949.

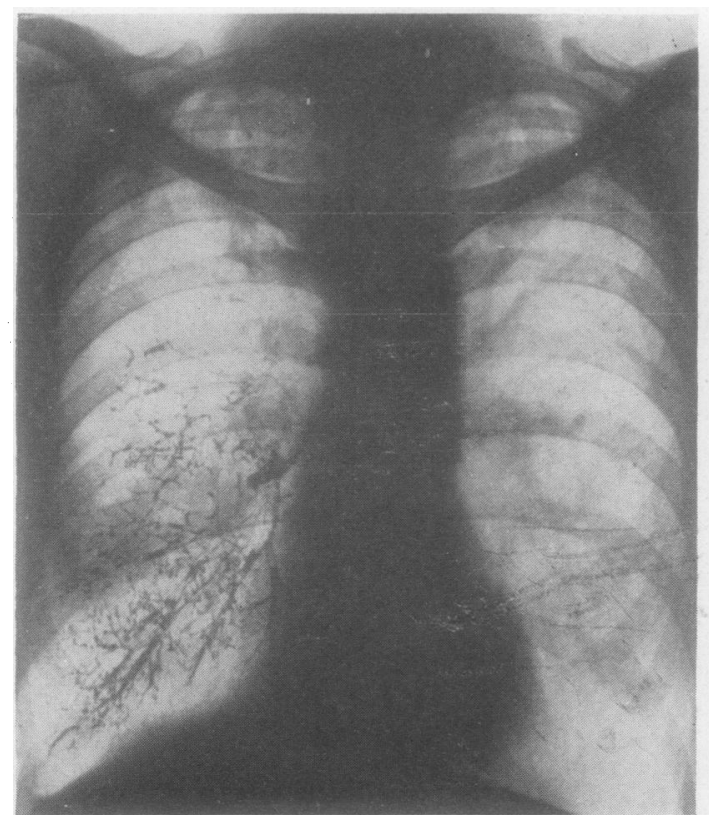

He was admitted to Harefield Hospital on Januar 31 , 1949, when a radiograph showed fibrosis an calcification in the right upper zone, pleural thickere ing at both bases, especially the right, and consolida tion in the lateral segment of the middle lobe. Sp4 tum on repeated examinations was negative fơ tubercle bacilli.

Bronchoscopy, as an out-patient, on December 1948, had shown nothing abnormal. On March $\varpi_{0}$ 1949 , after admission, a second bronchoscopy showe a soft, nodular mass in the lateral branch of the middle lobe bronchus; biopsy of this showed necrotic tissue only. On March 22, 1949, a third bronchoscopy wass reported to show a normal middle lobe orifice, this lesion seen at the second bronchoscopy being no longer visible.

On March 31, 1949, bronchograms showed a block of the middle lobe bronchus (Figs. $6 a$ and $b$ ).

On April 22, 1949, at thoracotomy a firm mas occupying most of the middle lobe was felt, and the right lung was removed.

The pathologist's report on the specimen was follows :

"The lateral division of the middle lobe bronchurf is occluded by growth. This measures about $1.2 \mathrm{cntu}$. in transverse diameter, and extends into the laterab segment for about $3 \mathrm{~cm}$. A snout of growth is presert in the lumen of the middle lobe bronchus so that the medial segment is also obstructed. The lobe shrunken, fibrosed, and shows lipoid pneumonitio The scar at the apex of the lung is calcified.

"Polygonal-celled carcinoma; the apical scar cor tains calcified caseous foci which have been part replaced by bone with marrow."

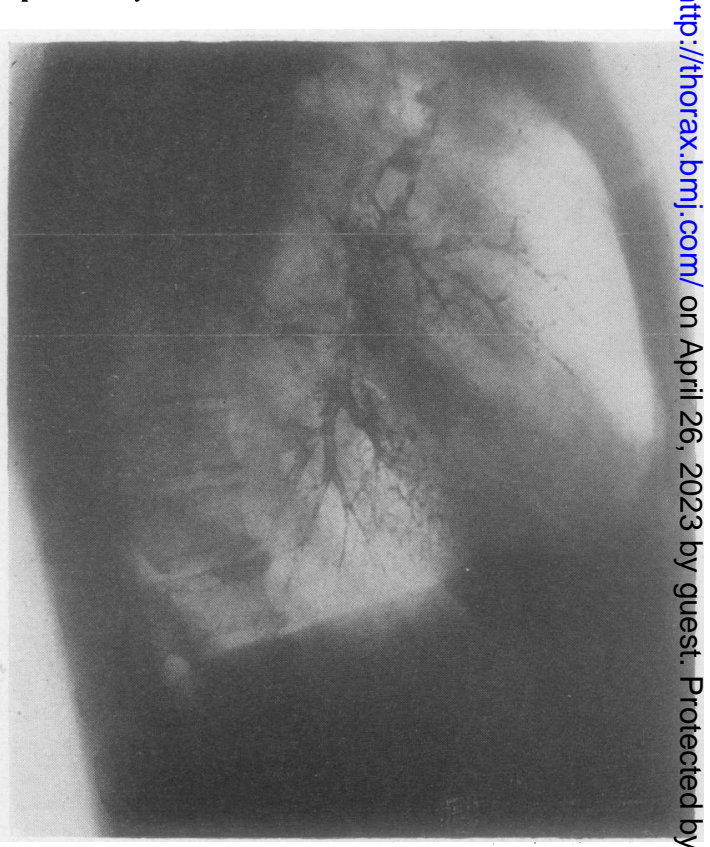

Figs. $6 a$ and $b .-$ Bronchograms showing obstruction of the middle lobe bronchus. This obstruction was proved later to be due to carcinoma arising at this site. Fig. $6 a$ also shows calcification at the apex of the right lung. 


\section{THORACOTOMY}

As the diagnosis may be uncertain before thoracotomy in these cases, the records of all patients over the age of 20 who, in the five-year period under review, had had a middle lobectomy performed for bronchiectatic changes not associated with malignant disease were analysed in an attempt to determine any difficulties that might have arisen in excluding carcinoma. There were 16 such cases. Eight of these had bronchiectasis in other lobes or segments, and therefore presented no diagnostic difficulty. It is of interest that in only one of these was calcification at the hilum demonstrable in plain radiographs. The remaining eight included three cases which showed radiographic evidence of hilar calcification, and because of this a presumptive diagnosis of post-tuberculous broncho-stenosis had been made.

The other five cases had no such indication of their aetiology, and therefore will be considered more fully.

Case 1.-A man aged 31 had had cough and $2 \mathrm{oz}$ $(60 \mathrm{ml}$.) yellow sputum daily for two years and recurrent brisk haemoptyses for eight months before admission to Harefield Hospital. A radiograph at this time showed collapse and consolidation of the middle lobe. Sputum was repeatedly negative for tubercle bacilli. Bronchograms showed gross bronchiectasis, and because of this the lobe was removed.

Case 2.-A woman aged 29 was well until April, 1947 , when she developed "influenza," followed by severe right-sided pleuritic pain, cough with copious foul sputum, and fever. A diagnosis of lung abscess was made, and she was treated with penicillin, with relief of symptoms. In July, 1947, cough and foul sputum recurred, and in August, 1947, she was admitted to Harefield Hospital. A radiograph showed consolidation and collapse in a portion of the middle lobe. Bronchoscopy showed oedema of the middle lobe orifice. After a further course of penicillin and postural drainage, bronchograms showed collapse and bronchial dilatation. Thoracotomy in October, 1947, revealed a collapsed, shrunken, tough middle lobe which was removed. This in section showed many irregular abscesses. Hilar lymph nodes were normal.

Case 3.-A man aged 33 was well until October. 1947, when after a shivering attack he developed a hacking cough and, one week later, right-sided pleuritic pain, with fever. In November, 1947, he began to cough up purulent sputum tinged with blood. He had been losing weight. $\mathrm{He}$ was then admitted to Harefield Hospital, and a radiograph showed consolidation of the middle lobe. Penicillin, $2,000,000$ units daily, resulted in a subsidence of fever and reduction of sputum to $\frac{1}{2} \mathrm{oz}$. $(15 \mathrm{ml}$.) daily. Sputum was negative for tubercle bacilli. Broncho- scopy showed no abnormality, but in spite of clinical improvement little change occurred in the radiograph. In January, 1948, bronchograms showed bronchial dilatation in the middle lobe. In February, 1948. thoracotomy revealed a collapsed infected middle lobe which was removed. The hilar lymph nodes were tough and fibrous.

Case 4.-A man aged 23 had "pneumonia" three years before admission, followed by cough and sputum every morning. Eighteen months later he developed severe pain in the right side of the chest, with increase of cough and sputum, which became tinged with blood. He was admitted to Harefield Hospital in December, 1949. A radiograph at this time showed displacement of the heart to the right, and bronchograms showed collapse and bronchiectasis of the middle lobe. Sputum was negative for tubercle bacilli. Thoracotomy revealed a collapsed middle lobe, which was difficult to remove owing to much scarring from old tuberculous glands.

Case 5.-A woman, aged 50, was well until September, 1950, when she had right-sided pneumonia. Cough and pain in the right side had persisted off and on since then. In March, 1951, bronchoscopy was reported to have shown a small bleeding growth in the anterior segmental bronchus of the right lower lobe. A biopsy was reported as showing "normal tissue." On admission to Harefield Hospital in April, 1951, a radiograph showed collapse and consolidation of the middle lobe. Bronchoscopy showed stenosis of the middle lobe orifice, but no growth was seen. Sputum was negative for tubercle bacilli. In May, 1951, thoracotomy revealed a consolidated middle lobe and a dense mass of lymph nodes around the hilum. Some of this mass was calcified, and it obviously resulted from old tuberculous infection. The lobe was removed.

These brief summaries show that in all but the first of these cases there was a very close resemblance in clinical features to some of the neoplastic cases already described; and although the disease proved ultimately to have an infective basis, there was no way of deciding this before operation. Even if the symptoms had not been severe enough to require surgical treatment, the similarity of the clinical picture to that produced by neoplasm of the middle lobe bronchus left no doubt of the necessity for thoracotomy.

Even at thoracotomy, much difficulty may be experienced in deciding the nature of the primary disease, as in both infective and neoplastic cases the tissues are often extensively adherent and the lymph nodes grossly involved. In fact, at times the diagnosis must remain uncertain until the specimen has been examined by the pathologist. To obviate this difficulty, Touroff (1949) has a frozen section examined at the time of the operation. In this way he has on several occasions been able 
to exclude malignant disease and confidently perform lobectomy rather than pneumonectomy.

\section{SUMMARY}

In the last few years much emphasis has been placed on the syndrome produced by collapse and inflammatory changes in the middle lobe secondary to stenosis of its bronchus by pressure, and sometimes by erosion by infected hilar lymph nodes. Although this is the common cause of the "middle lobe syndrome," carcinoma may arise from the middle lobe bronchus and cause an exactly similar clinical picture.

Nine cases of carcinoma arising from the middle lobe bronchus have been treated by resection at Harefield Hospital in the five-year period, July, 1946, to July, 1951. These cases are described and the manner in which they simulated those due to other disease processes, including lung abscess, interlobar empyema, and, above all, post-infective bronchostenosis, has been discussed. They constituted $5 \%$ of the total number $(185)$ of cases of bronchial carcinoma treated by resection duriog this period.

I should like to thank Mr. T. Holmes Sellors a Dr. L. E. Houghton for their encouragement a@d helpful criticisms given during the preparation of tofs paper ; Dr. L. G. Blair for his help and guidance in the radiological interpretation of these cases; M. E. F. Chin, Mr. T. Holmes Sellors, Dr. L. $\vec{E}$.

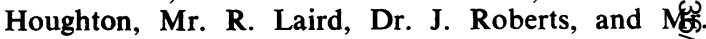
Vernon Thompson for permission to publish these cases; and Dr. D. M. Pryce for the pathologicall reports.

\section{REFERENCES}

Brock, R. C. (1943). Guy's Hosp. Rep., 82, 82.

- (1946). The Anatomy of the Bronchial Tree. London. (1950). Thorax, 5, 5 .

Cann, R. J., and Dickinson, J. R. (1937). Guy's Hosp. Rept, 87, 295 .

Carr, D., Skinner, E. F., Robbins, J. G., and Kessler, C. R. (1950) Dis. Chest, 17, 618.

Graham, E. A., Burford, T. H., and Mayer, J. H. (1948). Postgr@ Med., 4, 29.

Mason, G. A. (1949). Lancet, 2, 587.

Neuhof, H., and Copleman, B. (1940). Surgery, 7, 236.

Paulson, D. L., and Shaw, R. R. (1949). J. thorac. Surg., 18, 747.

Touroff, A. S. W. (1949). Ibid., 18, 758.

Woolner, L. B., and McDonald, J. R. (1950). Dis. Chest, 17, 1 o 